\title{
AN OVERVIEW OF CONSTANTINE'S TRAM SAFETY
}

\author{
Abdelaziz Kahlouche $e^{1}$, Rachid Chaib ${ }^{2}$ \\ ${ }^{1,2}$ Laboratory of transport engineering and environment, Department of transport engineering, \\ Frères Mentouri University, Constantine, Algeria \\ +213776560836,kahloucheaziz@yahoo.fr
}

\begin{abstract}
This paper presents an overview of the tram safety in the city of Constantine. The objective of this work is to contribute to the improvement of railway safety of a new urban transport mode in Algeria, whose safety culture is absent in the behaviour of citizens, in the face of major risks linked to the operating of Constantine's tram. In this context this article focuses on four parts: safety and risk management, railway risks, analysis of accidents, safety system and human factors (tram driver). This paper discusses the main issues, challenges and future directions of public transportation safety on these themes.
\end{abstract}

Keywords: Tramway of Constantine, risk assessment, safety, railway accident

\section{Introduction}

Transport plays a vital role in the economic and commercial development of countries and, consequently, in the well-being of their people. The transport system needs to be sustainable form an economic and social as well as an environmental aspect, to meet the demand of enlargement and sustainable development, but currently, the road transportation mode is being criticized more and more because of its major negative impact on the environment and the public health. Where the road traffic accidents have been and are continuing to be a major contributor of human and economic costs (Soltani and Askari, 2014). Therefore, for the prospect of sustainable development, the attraction for the railway transport has increased. Effectively, this main of transport has been considered one of the safest modes of transport in the world. Risk comparisons show that railway and air travel are the safest modes of transport per travelled passenger-kilometre (Silla and Kallberg, 2012). Therefore, its safety performance is always the public primary concern and required to be further improved by economic and political demands (An et al., 2006). Generally, urban railway transport accidents are material accidents that do not cause serious human damage, but they have a direct effect on the reliability of the transport system. Not only are disturbances on the main transport corridors degrading the network users, but more importantly, they have a negative impact on business and personal activities that depend on reliable programming (OECD, 2010). However, the effectiveness and the safety of railway operations depend on many factors including railway traffic rules, equipment reliability, general and safety management, and human factors. In particular with regard to human factors, it may be added that they are very important in the railway system just like in the case of any other complex system (Dhillon, 2007).

In Algeria one of the developing countries in northern Africa, the return to urban railway transport in big cities was an obligation for the authorities, due to the inability of the infrastructure of road transport and the bad quality of service (congestion, high travel time, discomfort etc.) faced with the great demand of transport in these cities.

Nowadays, the Algerian states have invested more than 40 billion dollars for the realization of the projects of transport in Algeria, including the realization of the trams in the big cities (ANDI, 2014). The operation of this means of transport has been awarded to the French partner [RATP Dev] in order to better transfer the European experience in the operating of this new mode of transport in Algeria. despite the fact that the safety management system adopted by the operating company was somewhat strict in terms of these safety procedures, but the difference in the behaviour of the European citizen and the Algerian citizen and supports the lack of a safety culture (Chaib et al., 2012) of the latter in the face of the risks associated with the operation of this mode of transport has contributed to the appearance of many accidents and incidents.

In this context the primary objective of this paper is to make an overview of the safety management system in Constantine tram, by the analysis of accidents and incidents by their type, and study the contribution of the human factor to these accidents, and finally propose general recommendations to improve the safety of this urban railway transport recently established in the city of 
Constantine. The materials in this paper are organized as follows: in the second section, a discussion of safety and risk management is given; the third section gives an overview of the analysis of risks linked to railway operation; in section 4, the case study area and tram accidents are described; section 5 presents the influence of the human factor on railway safety; section 6 gives recommendations, and a conclusion will be given in final section.

\section{Safety and Risk Management}

\subsection{Concept of risk and safety}

According to the generic standard (IEC 61508, 1999) safety is defined as "the absence of any unacceptable level of risk" whither the risk is a term that has been defined many times and in many ways. In (ISO 31000, 2009) risk is defined as the "effect of uncertainty on objectives". The probability of occurrence of a potential accident and the severity of the damage caused by this potential accident are the two components that identify the concept of risk. Therefore, to define the probability level of a potential accident, IEC 61508 proposes a set of categories, each of which is associated with a frequency range. Given the difficulty of estimating these frequencies, this quantitative / qualitative association is favourable. Similarly, IEC 61508 defines the level of severity by associating the quantifications with the consequences generated by the potential accident. Indeed, the level of acceptability of risks is identified and evaluated using the Occurrence / Gravity matrix.

\subsection{Safety Management System}

A Safety Management System (SMS) is a management model that groups functions, responsibilities, practices, procedures and processes for risk prevention. Real and effective application of an SMS allows compliance with an extensive legal framework including guarantees of effective protection for persons and continuous improvement of health and safety conditions (Granerud and Rocha, 2011).

\subsection{Risk management process}

Risk management is the process of identifying, assessing and prioritizing risks relating to the activities of an organization, whatever the nature or origin of those risks, in order to deal with them methodically in a coordinated and economic manner, in order to reduce and control the probability of feared events, and to reduce the possible impact of these events. According to the standard (ISO 31000 , 2009) risk management refers to a coordinated set of activities and methods that is used to direct an organization and to control the many risks that can affect its ability to achieve objectives. The Figure 1 (Yatskiv and Gromule, 2016) explains the risk management process, which need to realise on systematic approach.

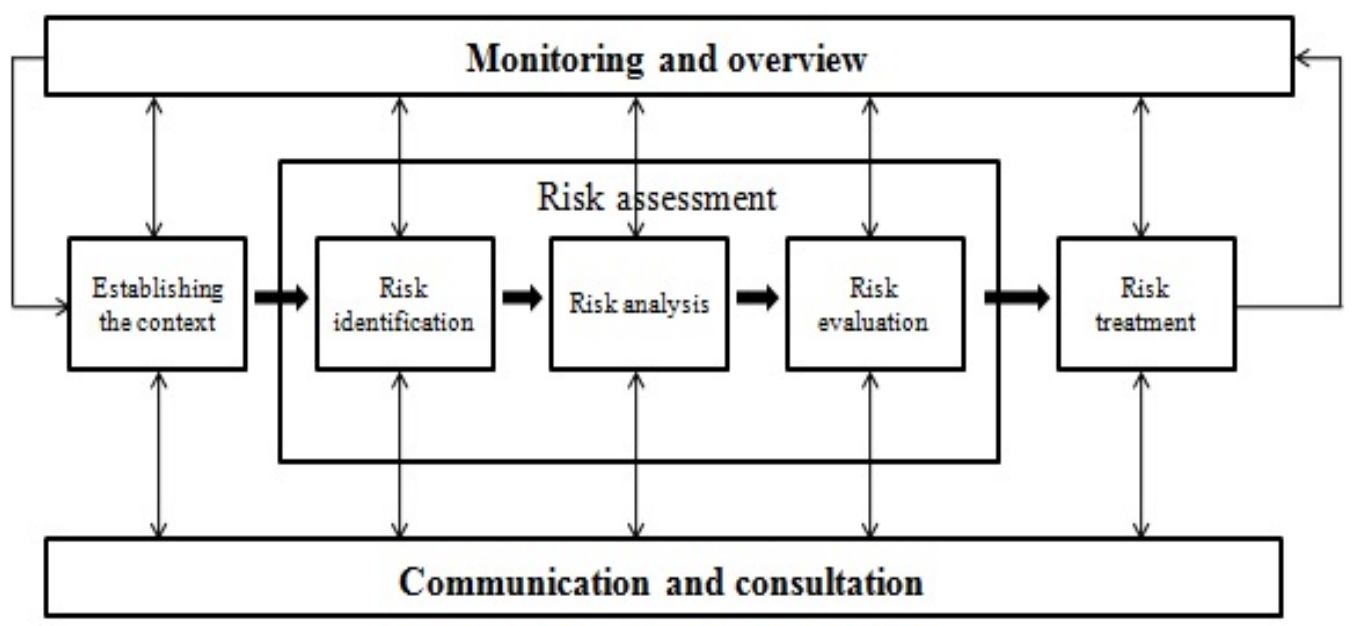

Figure 1. Risk management process 


\section{Analysis of Risks Linked to Railway Operation}

\subsection{Risks process}

The risks existing in the railway system can affect either a unique individual (individual risk), or several people (collective risk), or the system (rolling stock and infrastructures), or the environment. Generally, we distinguish three categories of accidents: the accidents user, the accidents system and the accidents user / system. This classification can be completed with the category accident environment / system (Hadj-Mabrouk et al., 1998). The Figure 2 details the various accidents in the railway system, classified according to the categories above.

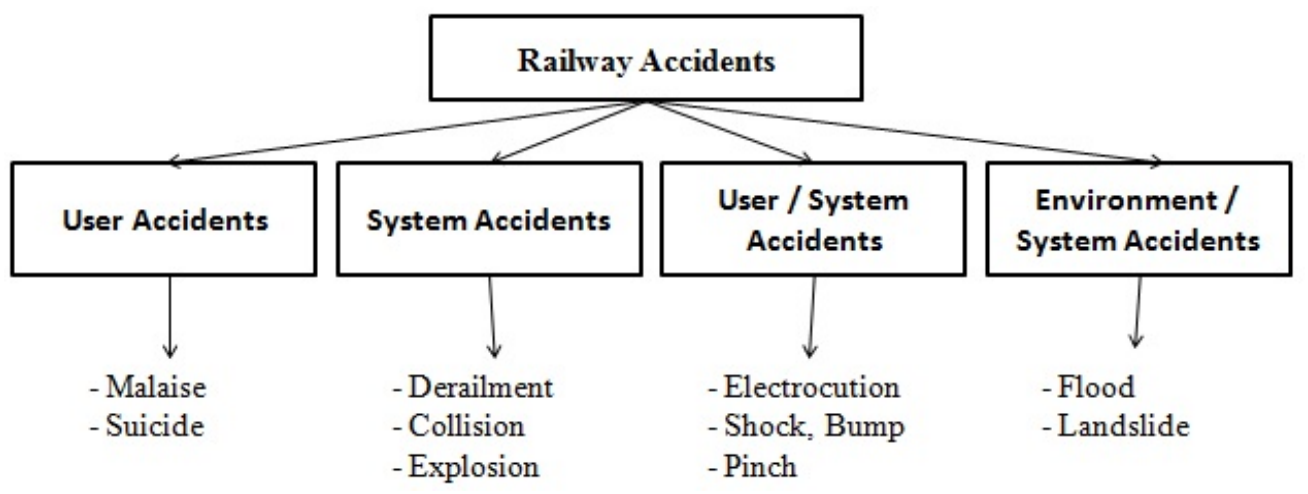

Figure 2. Classification of the various accidents in the railway system

The four categories in Figure 2 are described as follows:

User Accidents: They are associated with the damage caused to one or more users located within the system when no problem of operation of the system has occurred and no action of this user or users has been detected.

System Accidents: They are associated with damage to the system and to users or personnel in an accident initiated by the system itself.

User/ System Accidents: They are associated with the damage caused to one or more persons within the system, in the course of an action performed by the user or users during the normal operation of the system.

Environment /System accidents: They are associated with damage to the system and users during environmental conditions known as natural disasters.

\subsection{Risks assessment in railway system}

Risks assessment is a crucial step in the approach to preventing railway risks. It involves using different methods to reduce the risk so that it will be acceptable (Arena et al., 2015). The risk assessment in the railway system is based on the following steps:

- Hazards identification

- Causes/Consequences analysis

- Estimation of frequency/gravity

- Integration of prevention and protection measures

- Verification of the acceptability of the level of the risk

\subsection{Normative framework of railway safety}

In railway transport system, safety is ensured by the use of various systems which have been tested and proven efficiently for several years and are considered as guarantors of the safety of the transport system as a whole. The implementation of a system in the field of railway transport, whether urban or non-urban, is linked to the implementation of the reference system (EN 50126, EN 50128, and EN 50129), whose the main objective is to improve the health and safety performance of the railway systems and thus facilitate the reduction of risks to passengers and to preserve infrastructures (IEC, 2000). 
- EN 50126: Railway applications: the specification and demonstration of reliability, availability, maintainability and safety.

- EN 50128: Railway applications: communication, signalling and processing systems software for railway control and protection systems.

- EN 50129: Railway applications: communication, signalling and processing systems - safety related electronic systems for signalling.

\section{Analysis of Tram Safety in the City of Constantine}

\subsection{Presentation of Constantine's tramway}

The Tramway transport system of Constantine comprises a double-track line with a length of about $8 \mathrm{~km}$ and comprises 10 stations, Figure 3. The poles of bus exchanges and cultural centres are taken into account in the design of the system (Alstom, 2008). The tramway of Constantine serves urban, suburban and being urbanized areas, with an average of 24000 passengers per day, it aims to improve the offer of transport in the city by promoting a sustainable mobility.

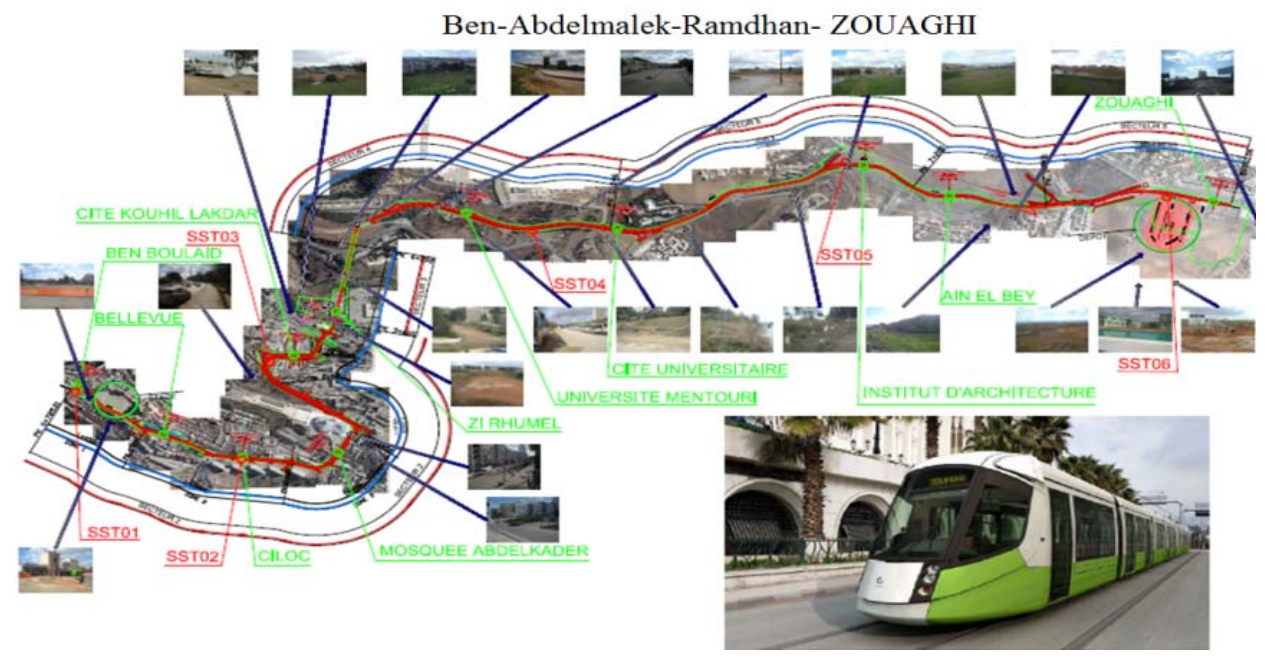

Figure 3. General view of the Constantine tramway line

\subsection{Incidents analysis}

Analysis of incidents recorded during the operation of the tramway, is a fundamental element in understanding the causal elements and their subsequent control. Effectively, improving the quality of incident analysis, allow the detection of fragility points to prevent incidents. In order to a better evaluate the level of safety of the Constantine tramway, the Figure 4 shows the number of incidents during the period (June 2016 - December 2016) which were avoided either by an emergency brake triggered by the tram driver or by the emergency stop button triggered by a traffic supervisor.

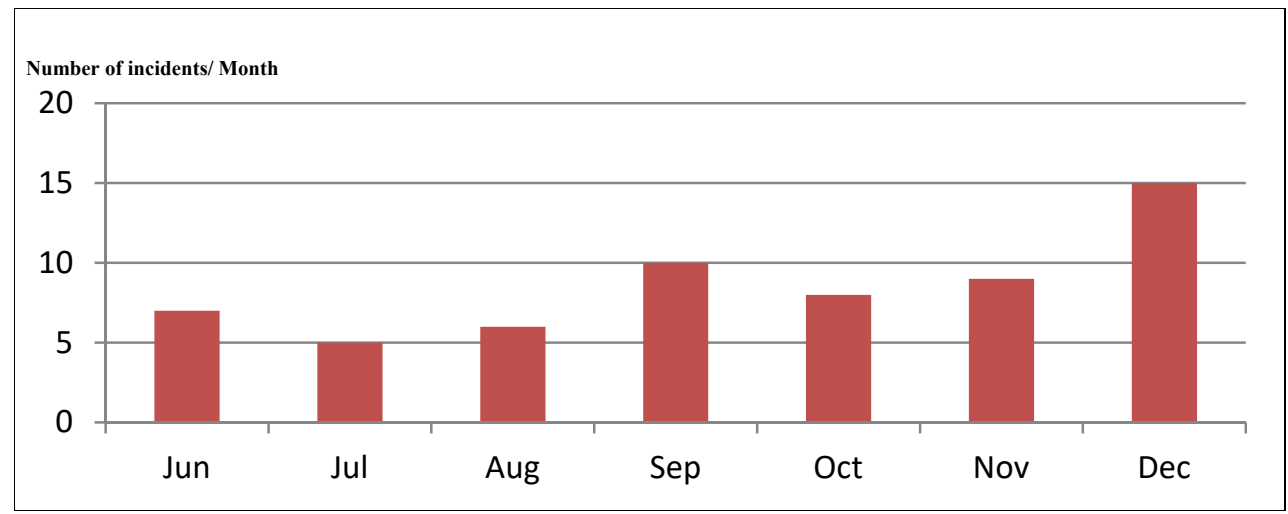

Figure 4. Statistics of incidents (June-December 2016) 
Analysis of incidents according to specific time periods considers the influence of time variable on tram accidents. The grave above shows that there is a small proportion of incidents occurred during the summer, because in those months the urban street network recorded smallest traffic volumes of both private cars and pedestrians. Autumn moths generally recorded highest traffic volumes because of the social re-entry (students, pupils and workers) in the city, and as a consequence, the highest proportion of incidents. This shows that additional safety measures need to be implemented in order to lower those situations in this period.

\subsection{Analysis of accidents linked to tram operation}

According to a qualitative and quantitative analysis of tram accidents whose primary source is the SETRAM Company responsible for operating the Tramway line; different scenarios were recorded. Remember that the tram accidents occur despite the safety measures taken by the operator and some accidents with light vehicles and pedestrians have serious consequences (Kahlouche and Chaib, 2017). Since its insertion in July 2013 until December 2016, its operation was not without adverse effects on the city of Constantine, tram accidents with road users continue to occur, and several collisions were recorded of different severities.

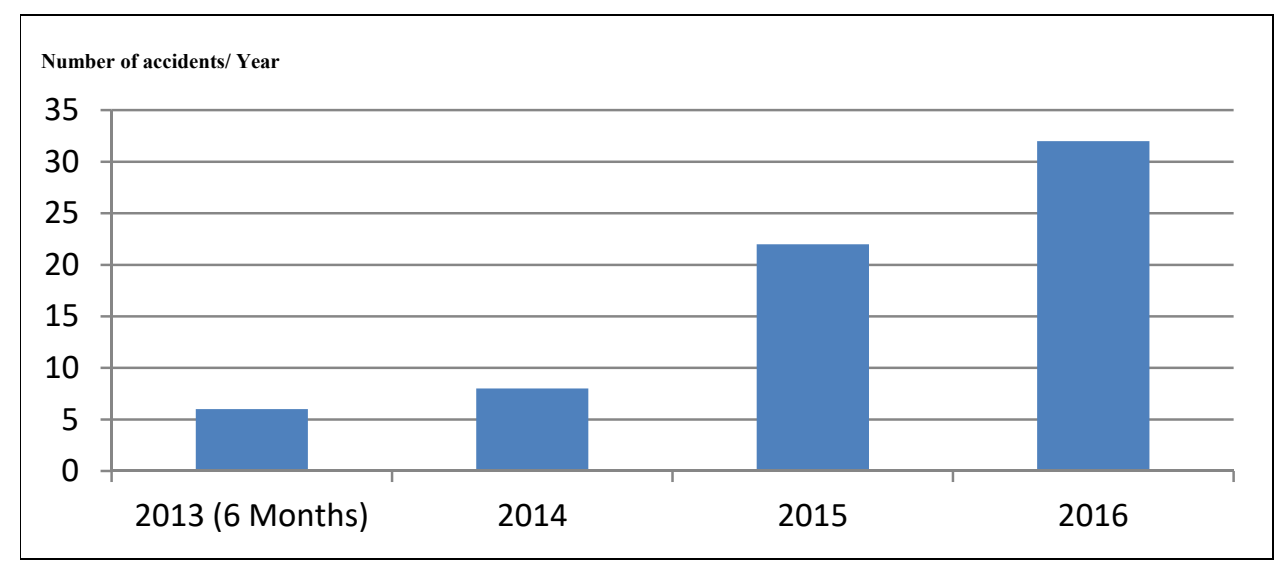

Figure 5. Distribution of number of accidents per year

By observing absolute number of tram accidents for the period from 2013 to 2016 (Fig. 5) it can be observed that there is a significant increase in the number of accidents each year, despite the awareness campaigns and actions taken by the operator in order to sensitize citizens about the risks of the tramway, which implies the existence of other causes that affect tram safety, and which will be presented in this article.

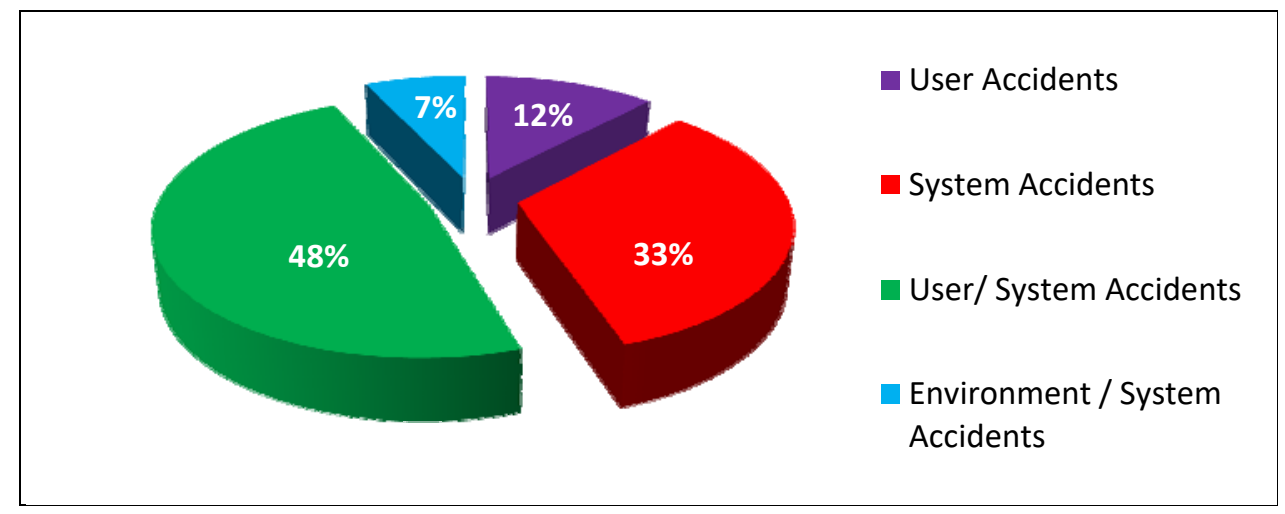

Figure 6. Distribution of accidents by category

Figure 6 shows the distribution of Constantine tramway accidents by category. It can be seen that the most of accidents are of the user / system type, which means that all events causing accidents occur 
with the interaction of the human factor and the system. So it is essential to put a plan of actions that takes into consideration the human factor in these automated systems in order to achieve a high level of safety.

\section{The Influence of the Human Factor on Railway Safety}

With the improvement of technical reliability, the current trend is to attribute the malfunctions of the systems, generators of accidents, to a mistake of the human operator. The operator is considered as weak point of the system and limit of performance and safety. Thus, human error is a major causal factor in the emergence of accidents in several safety sectors, including railway transport (Hadj-Mabrouk, 2010). Over the years, a large number of railway accidents resulting in many fatalities and a high economic cost have occurred due to human factors-related problems in the design and operation of railway systems around the world (Dhillon and Balbir, 2007).

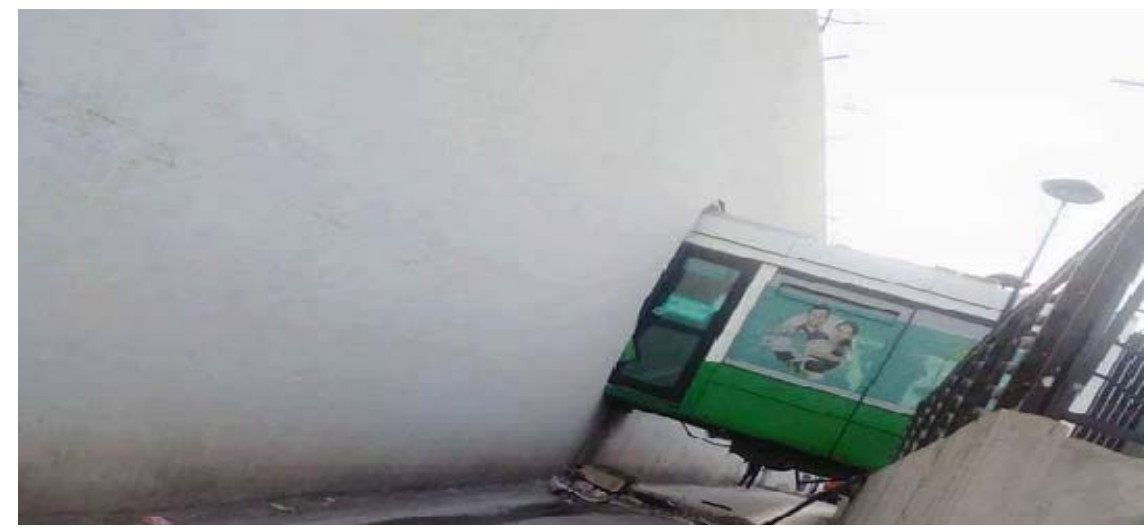

Figure 7. Constantine tramway accident due to human error

Figure 7 represents a system accident that happened at the Constantine tramway on August 16, 2016 due to driver error. The human factor have a great influence on railway system's safety, in the tram operation the driving is a complex task, placing a high demand on the driver's knowledge, experience and attention. The driver is required to drive safely according to traffic rules and conditions (Naweed and Rose, 2015), but he may be subject to errors such as failure to observe the signals displayed, exceeding the permitted speed, or disrespect the procedure, all these errors are a symptom of a poor work organization, poor or inadequate training of drivers, and may adversely affect the operation of this system under optimal safety conditions.

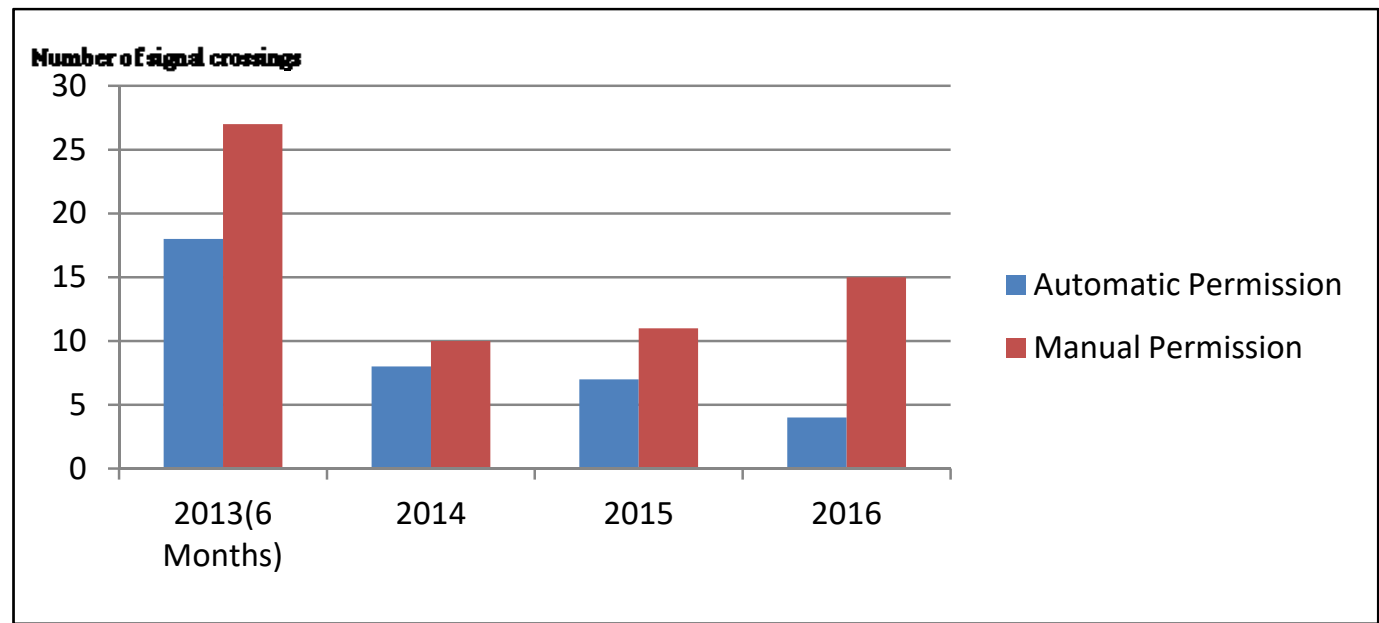

Figure 8. Report of passed restrictive signal by Constantine's tram drivers

Figure 8 shows the number of signal crossings recorded over the $8 \mathrm{~km}$ of the Constantine's tram line, with the exception of 2013, which was a period of mastery of this new mode of transport. There is an 
increase in the number of manual signal crossings which implies that drivers in the Constantine's tram were subject to high levels of fatigue because of the hypo vigilance (Hadj-Mabrouk, 2016) due to the schedules of driving ( 7 hours) and the wrong citizen's behaviour in the stations or in the intersections. In order to improve the human reliability and reduce its errors we suggest that:

- Managers should reduce the workload of Constantine's tram drivers; arrange an appropriate work/rest schedule (Tsao et al., 2017).

- Improve the physical working environment for the drivers.

- Planning sessions of psychomotor vigilance test for the drivers.

- Periodic medical examination.

\section{Recommendations}

\subsection{General recommendation}

\section{Urban Traffic Control Systems}

Drivers' behaviour in intersections crossed by the tram is not only influenced by the rules of the tramway priority in the intersection but also by the road features that influence right or wrong behaviour of the driver (Moutchou et al., 2014). The majority of intersections with the tram platform are managed by standard traffic lights systems enabling to give priority to the tramway, but since its implantation in April 2015, some signals don't work properly e.g.: The traffic light stays in red but no tramway is travelling in the intersection or the signal gives the permission to the road users with tramway on the intersection.

Possible improvements: Using the method of active priority or dynamic priority, this method consists in modification of the intersection signals to authorize the passage of the tramway which has been detected. The advantage of this method is that it is not constrained by a traffic light fixed cycle (Bhouri et al., 2015).

\section{The awareness campaigns}

Effective awareness campaigns are an essential element in improving safety in the railway system, in order to create awareness among the passengers about safety guidelines that they need to follow while on tram and on platforms. At the Constantine tram, there is a lack of planning of the awareness campaigns by the operating company; the process was limited only when the operation started, so it is essential to continue the awareness campaigns to increase the safety culture in the society.

\subsection{Recommendations for the second line}

The first line is a reference on which the existing transport network of the city of Constantine must continue to evolve today. In order to link it with the new urbanization area, the tramway of Constantine will benefit from a second line consists of $10.4 \mathrm{~km}$ and 11 passenger stations. One of the main ways to make this line as safe as possible is a good design of its urban insertion, considering three main aspects: the visibility between the tram and other street-users (road vehicles, pedestrians), the perception of the system (and information to other street users) and the tramway protection in its interaction with them (Fontaine et al., 2016).

\section{Conclusion}

In this work, an analysis of the safety of the Constantine tramway has been made. The objective has been to contribute to the improvement of railway safety of a new urban transport mode in Algeria. We can say that accidents depend not only on the tramway environment but also on the safety management system established by the operating company which must be revised taking into account the coherence between this automated system and the human factor. The results of this study could be used to suggest a safety management system focusing not only on the technical parts but also on the underlying human factor factors. Finally, it can be said that we must now live in harmony with the tramway in operation, for this purpose, it is necessary to initiate educative and preventive as well as promotional activities, which would inform, train and encourage desirable behaviour of citizens in order to achieve a high level of safety culture. 


\section{References}

1. Alstom Transport. (2008) General description of the transport system 'Tramway of Constantine'.

2. An, M., Lin, W., Stirling, A. (2006) Fuzzy reasoning-based approach to qualitative railway risk assessment. Proc. IMechE, Part F: J. Rail and Rapid Transit, 220 153-167.

3. ANDI. (2014) National Investment Development Agency Algeria. www.andi.dz

4. Arena, M., Foiadelli, F., Acquaro, G. (2015) Functional safety of railway systems, AEIT International Annual Conference (AEIT), DOI: 10.1109/AEIT.2015.7415248

5. Bhouri, N., Mayorano, F., Lotito, P., Haj-Salem, H., Lebacque, J. (2015) Public Transport Priority for Multimodal Urban Traffic Control. Cybernetics And Information Technologies, 15(5) 17-36, ISSN: $1314-4081$

6. Chaib, R., Verzea, I., Benidir, M., Taleb, M. (2012) Promoting a culture of health and safety at work: safety - a permanent priority, WIT Transactions on Information and Communicatio $n$ Technologies, 44, 405-413, doi:10.2495/RISK120341

7. Dhillon, B.S. (2007) Human Reliability and Error in Transportation Systems, Springer-Verlag London. DOI: 10.1007/978-1-84628-812-8

8. Fontaine, L., Novales, M., Bertrand, D., Teixeira, M. (2016) Safety and operation of tramways in interaction with public space: 6th Transport Research Arena; Transportation Research Procedia 14 1114-1123.

9. Granerud, R.L., Rocha, R.S. (2011) Organisational learning and continuous improvement of health and safety in certified manufacturers. Saf. Sci. 49(7), 1030-1039. http:// dx.doi.org/10.1016/j.ssci.2011.01.009

10. Hadj-Mabrouk, H., Stuparu A. and Bied-Charreton D. (1998) Example of a typology of accidents in the field of guided transport. General Review of Railways. 17-55.

11. Hadj- Mabrouk, H. (2010) Introduction to safety and the analysis of technological and human risks, 3rd International Symposium on Maintenance and Risk Management, Apr 2010, Rabat, Morocco.

12. Hadj-Mabrouk, H. (2016) Contribution of chronobiology vigilance in public transport safety, International Journal of Multidisciplinary Research and Development, 3(9), 214-221. ISSN: 23494182

13. IEC 61505. (1999) Electrotechnical Commission, functional safety of electrical/ electronic/programmable electronic safety-related systems.

14. IEC. (2000) International Electrotechnical Commission, functional safety of electrical/ electronic/programmable electronic safety-related systems.

15. ISO 31000. (2009) Risk management, Principles and guidelines.

16. Kahlouche, A., Chaib, R. (2017) Analysis of tram safety: Case study of Algeria. Procedia Engineering, 178, 401-408. DOI.org/10.1016/j.proeng.2017.01.076

17. Moutchou, F., Cherkaoui, A., El Koursi, E. (2014) Factors Influencing Driver's Behavior at Intersections Crossed By the Tram. 3rd International Conference on Road and Rail Infrastructure; Road and Rail Infrastructure III, 785-792.

18. Naweed, A., Rose, J. (2015) "It's a frightful scenario": A study of tram collisions on a mixed traffic environment in an Australian metropolitan setting: 6th International Conference on Applied Human Factors and Ergonomics; Procedia Manufacturing 3 2706-2713.

19. OECD (2010) Improving reliability on surface transport networks, Editions OECD. ISBN 978-92-8210241-1

20. Soltani, A., Askari, S. (2014) Analysis of intra-urban traffic accidents using spatiotemporal visualization techniques. Transport and Telecommunication, 15(3), 227-232. DOI 10.2478/ttj-20140020

21. Silla, A., Kallberg, V. (2012) The development of railway safety in Finland, Accident Analysis and Prevention, 45, 737- 744 .

22. Tsao, L., Chang, J., Ma, 1. (2017) Fatigue of Chinese railway employees and its influential factors: Structural equation modelling, Applied Ergonomics, 62 132-141. DOI.org/10.1016/j.apergo. 2017.02.021

23. Yatskiv (Jackiva), I., Gromule, V. (2016) Holistic Approach to Passenger Terminal Risk Estimation. IEEE, 643-649, DOI 10.1109/SMRLO.2016.114 